\title{
Remote Laboratories - A Cloud Based Model for Teleoperation of Real Laboratories
}

\author{
http://dx.doi.org/10.3991/ijoe.v9i2.2491 \\ C.M. Markan ${ }^{1}$,Satendra Gupta ${ }^{1}$, Sajal Mittal ${ }^{2}$ and Goutam Kumar ${ }^{1}$ \\ ${ }^{1}$ Dayalbagh Educational Institute, Dayalbagh, Agra, India \\ ${ }^{2}$ Indian Institute of Technology, Kanpur, India
}

\begin{abstract}
Remote Laboratories provide access to real experiments without time and location restrictions with necessary guidance required to perform experiments. Remote laboratories have emerged as an alternative to real laboratories and have shifted the past pedagogy from faculty-centric to student-centric approach. Over the past decade several remote laboratories have emerged in different parts of the world including those developed under Government of India's National Mission on Education through ICT as part of Right to Education (RTE) Program. Various approaches have been implemented to promote the use of remote laboratories in higher education. Technology to build remote laboratories has evolved considerably, there is still a growing concern regarding the multi-user scalability of these remote laboratories. Also, there is a need to incorporate innovative ideas such as collaboration of resources among universities within the remote laboratories framework. In this paper we propose a cloud based architecture for remote laboratories that provides scalability, more efficient use of resources, lower cost of ownership and shorter production time. Implementation of an Analog Communication lab, based on this architecture, has also been described. Advanced web technologies have been used to design an interactive user interface that makes teleoperation of high end instrumentation more convenient.
\end{abstract}

Index Terms-Remote Laboratory, Cloud based Architecture, Emona-DATEx, Ni-Elvis, Analog communication

\section{INTRODUCTION}

It has been shown that transition from a Faculty-Centric to a Student-Centric teaching approach can bring about many positive changes in the education system e.g. enhanced learning, independent thinking and creativity in students [1].Remote Laboratories, that favor the studentcentric approach, have clearly shown their academic usefulness [3] and have emerged as a possible substitute to real laboratories[4].

There has been immense growth in the area of Remote Labs in the past decade. Many remote labs including iLabs MIT, NetLabs UniSA [15], VISIR [17], WebLabsDeusto [16], and eVALIDATE [9] have been developed worldwide. Many universities have included experimentation on remote labs as part of their curriculum. NetLabs, VISIR, and WebLabs have developed their remote labs that provide interface for electronics experiments based on analog circuits. iLabs [20] have developed a shared architecture using a service broker that provides a methodology to share remote labs experiments among campuses of different universities [19]. iLabs architecture is designed to focus on faculty-centric approach, however, our aim remains to move from faculty-centric approach towards a student-centric approach. Besides these, several other remote labs [21] have been developed that provide user interface for performing complex experiments and focus on various other areas [22] including Analog Communication [8].

Virtual labs that provide access to simulators are also available. While these labs have the advantage of scalability, there are situations when simulators work against fundamentals. Therefore, their results are not always accurate. In such situations, remote labs prove to be more advantageous over virtual labs.

Many remote labs have automated processes and have a degree of programmability however most of them are not designed to handle concepts similar to multitasking or multithreading and hence are not ready to reap benefits of resource sharing. One of the major technological hurdles in this direction is to introduce the concept of 'multi-user scalability' [6]. Multi-user scalability implies building a mechanism wherein many users work on the same hardware at the same time with each getting the feel of ownership or an 'illusion of parallelism'. It may be stated here that technically most electronic equipment are not multiuser and have to be controlled by one user at time. However with introduction of modern high speed switching and data acquisition technology it is possible to conduct some experiments in a multitasking or multithreaded way.

Remote labs use the concept of cloud computing [14]. Cloud computing is a new paradigm that provides many features with regard to efficient management of computing infrastructure. It provides a model for enabling convenient and on-demand network access to shared configurable resources that can be rapidly provisioned and released with minimum service provider interaction. The concept of cloud computing emerged when Mainframe Computers with high cost and greater computation power became available and it became important to find means to share their computation power in order to justify their cost. Thin-clients were put to use to provide access to multiple users. In a similar way, remote labs were developed to provide shared access of high-end instruments to multiple users simultaneously using internet. By introducing more advanced concepts like virtualization scalability of virtual labs can be further enhanced.

Despite the advantages of remote labs, they still face certain technological hurdles that belittle the potential applications for which they were created. One of them is that only a single user can access and control an instrument throughout the duration of the experiment in what is 
termed as one-equipment-one-user 'interactive mode'[7]. In this mode assuming that an experiment needs a one hour slot we can expect only a single user or a group of collaborative users will have access to the lab in that duration and hence no more than 24 such users would complete an experiment in a day. Another approach is the 'batch mode' comprising of a queuing mechanism to handle multiple user's requests simultaneously. Execution time in this mode plays an important role influencing the overall time of experiment. Our approach is very similar to iLabs. We have implemented a cloud based methodology utilizing virtualization, to build our remote lab for Analog Communication that helps in reducing the overall time for performing an experiment.

Analog Communication is an important part of the engineering curriculum [2]. Remote labs for analog communication [12] require a more comprehensive approach and design of back-end infrastructure, because there should be lesser interaction of the hardware with the environment so that noise can be minimized.

Analog Communication experiments like Modulation, Demodulation etc., are traditionally conducted using a breadboard and electronic components. We have used Emona DATEx board (Plug-in module for Ni-Elvis) and National Instrument's Educational Remote Instrumentation Suite (Ni-Elvis) to provide access of analog communication experiments. Emona DATEx board provides a block level approach to telecommunication experiments which is more prevalent for engineering students. Although the DATEx-Elvis II bundle [8] provides excellent engineering communications concepts, it does not provide autonomous process to perform these experiments over internet.

To provide students a complete e-learning environment that includes information on theoretical concepts and experimental procedure, a website (http://www.evalidate.in) as shown in Figure 1 has been developed. Video Tutorials, that provides step by step procedure to perform experiments, have also been made available. Questions, related to experiments, have also been provided for self evaluation.

To give a traditional feel to Emona DATEx board in Remote Lab environment, a frontend has been developed that provides students the flexibility to perform any communication experiment using Emona DATEx blocks. In this paper in section II we describe different modes of operation of remote lab. In section III we describe the architecture and front-end of the remote laboratory based on Emona-DATEx and Ni-Elvis set up. In section IV we describe backend data flow operation. In section $\mathrm{V}$ we discuss about sustainability and scalability. In section VI

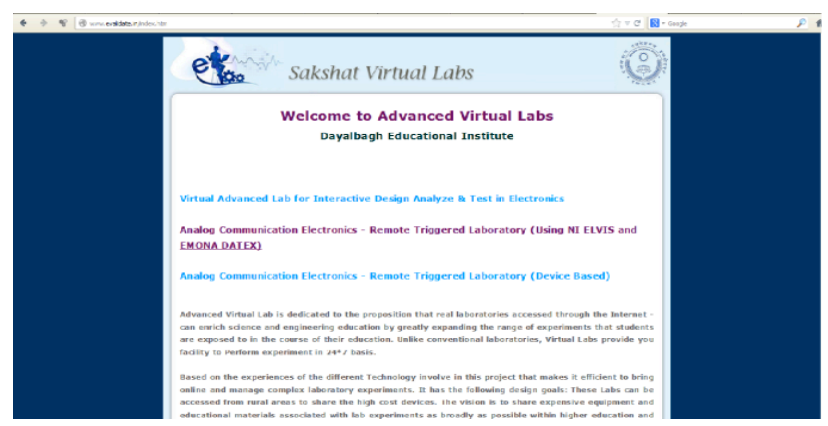

Figure 1. Picture of the website containing supplementary material for experiments we examine the remote lab experiment results by comparing them with traditional physical lab experiment results. In section VII we bring forth the differences between our lab and other remote labs.

\section{MODES OF OPERATION}

The following steps have to be undertaken to perform any lab experiment (i) Understanding the objectives and theoretical foundations, (ii) Planning, designing of experiment and preparing instrument settings (iii) Executing on hardware and data acquisition (iv)Data analysis and report writing.

\section{A. Interactive Mode}

Most Remote labs operate in interactive mode in which hardware is booked for one hour for one student. If a student needs to do 10 labs a semester, then every student needs 10 hours per day for 6 months. That implies at most 150 students can perform experiments using remote labs in every semester. This is not very promising given the fact that remote labs cost nearly 10-100 times more than a real lab. If remote labs need to address a larger population then the only solution would be to replicate more hardware. We therefore need to take a fresh look at the way we perform experiments and explore new ways to introduce scalability into remote labs [10].

\section{B. Batch Mode}

We explore two different methods (i) Reduce hardware interaction time or reduce slot duration (ii) Introduce oneshot hardware step to complete experiment in 'batch mode' [5].

Method 1: Reduce Slot duration. Out of the steps that must be undertaken to perform any experiment, as mentioned above, steps (i), (ii) and (iv) are cognitive tasks and must take their own natural time. However, execution and data acquisition, which involve the real hardware require only a fraction of total experimental duration and hence can be accomplished quickly. To reduce slot duration step (i), (ii), and (iv) needs to be decoupled from hardware backend i.e. step (iii). If this can be done then it is possible to reduce the slot duration to say 10 minsin a $1 \mathrm{hr}$ experiment. This basic step can improve scaling 4-5 times (assuming there is no need to redo). Though this method improves scaling compared to a real lab, yet it is not good for a classroom where multiple users still have a long waiting time to get a hardware execution slot.

Method 2: One-Shot Hardware execution \&Queuing: This method aims at speeding up hardware execution time by sacrificing the direct remote triggered human interactivity. In this mode a client works on an animated/simulated lab interface similar to method 2 to interactively (i) configure the instruments, (ii) setup experimental hardware and execute and (iii) record the observations. Working interactively on this animator/simulator, the user generates a command summary (somewhat similar to SPICE code) consisting of sequential commands for wiring the circuit netlist, instrument settings and data recording instructions.

As a result, the command summary generated on the animator/simulator is executed on the real hardware in a rapid 'one-shot' batch mode [10] on the remote triggered hardware backend. This implies that the circuit gets wired up on the relay matrix, input is applied to the circuit and the requested data is acquired by the oscilloscope and 
transferred to the client computer in one quick step. The acquired data is then played on an animated instrument at the client end for analysis and report generation.

\section{LAB ARCHITECTURE}

In order to perform communication experiments using the Elvis II-DATEx setup remotely, there is a need to eliminate the user interaction in order to perform the required connections for the DATEx modules as well as to provide extendibility to wiring circuits from the clientside. This is accomplished by using an Agilent LXI switching matrix. The Client-Server Architecture as shown in Figure 2 is a cloud based platform developed for Ni-Elvis and Emona-DATEx. Other remote labs using NiElvis and Emona DATEx[ 8,12], lack multi-user scalability. Presented architecture is improvised as it uses the existing resources to build up a scalable model of remote labs using Elvis-DATEx bundle.

This architecture leverages virtualization technology to utilize the computational power of the servers running at the back-end and to overcome the problem of handling too many requests at a time. This approach also does away with the requirement of having LabVIEW run time engine on the client side. On the client side it only requires Javarun time engine which is mostly preinstalled as it is required by other applications as well.

This architecture provides a robust mechanism for acquiring and displaying data from Ni-Elvis-DATEx bundle. Instruments have been connected on a private network to ensure protection from any unauthorized access and safety for user data. An SSH server has been setup for communication between Linux based main server and Ni-Elvis data acquisition board connected to a windows system. ElvisDATEx setup is covered behind a reverse proxy server to restrict direct access to instruments.

Instead of providing access to LabVIEW Virtual Instrument's (VI) build, to control instruments, LabVIEW VI are converted into DLL (Dynamic Link Libraries).DLL files provide flexibility to the developers as they are easy to upgrade and there is no need for recompilation. These DLL files are then invoked inside $C$ language code written within Microsoft Visual Studio for compilation. The executable files generated inside MS Visual Studio can easily be invoked from command line with parameters passed in a file invoked through command line path.

A Queuing mechanism has been implemented that handles multiple client requests by forwarding them in FIFO (first-in first-out) manner. When multiple users simultaneously run any experiment, the queuing algorithm keeps track of these requests and sends them to the data acquisition board for execution on hardware one by one. Hardware execution time for each request remains 2-3 seconds. Data from the acquisition board is acquired using the DLL files and processed on the SSH server before it is sent to the main server. The data from the main server is delivered to the client computer on the animated oscilloscope graphical interface. This interface looks like a real oscilloscope interface and displays the plots of data acquired from the acquisition board giving the users a feeling of working on a real oscilloscope.Figure 3 shows the design of the animated oscilloscope. The oscilloscope GUI provides various options like auto-scaling and mathematical functions that can be applied to the waveforms.
This setup allows clients to obtain real experimental data by wiring up a circuit in the remote laboratory using graphical interface of a DATEx virtual breadboard and instruments all through remote access over the internet.

\section{A. Emona DATEx board}

The Emona DATEx trainer is a plug-in module for the Elvis platform .DATEx blocks can be used to create complex communication circuits using a simple bottom up approach of assembling different circuit blocks. DATEx provides different circuit blocks like Adder, RC Filter, Multiplier etc that are used in engineering curriculum for solving telecommunication circuits in mathematical equations. Figure 4 shows the picture of EmonaDATEx Board set up in our laboratory. Students can log in and perform any experiment by simply wiring up circuit on the GUI.

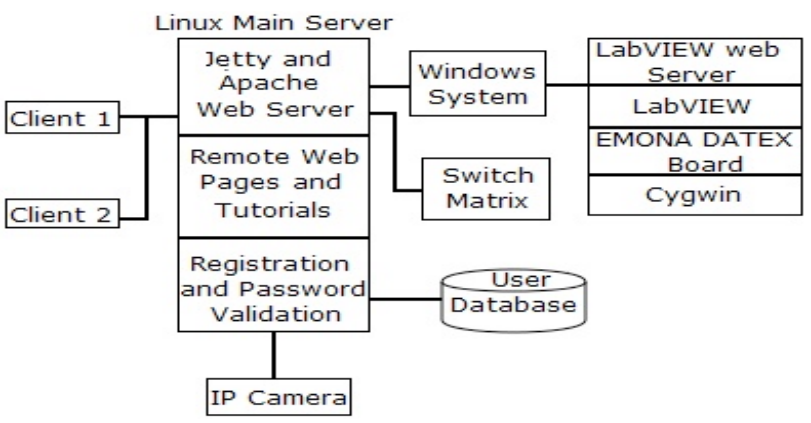

Figure 2. Client Server Architecture of Remote Laboratory For Analog Communication

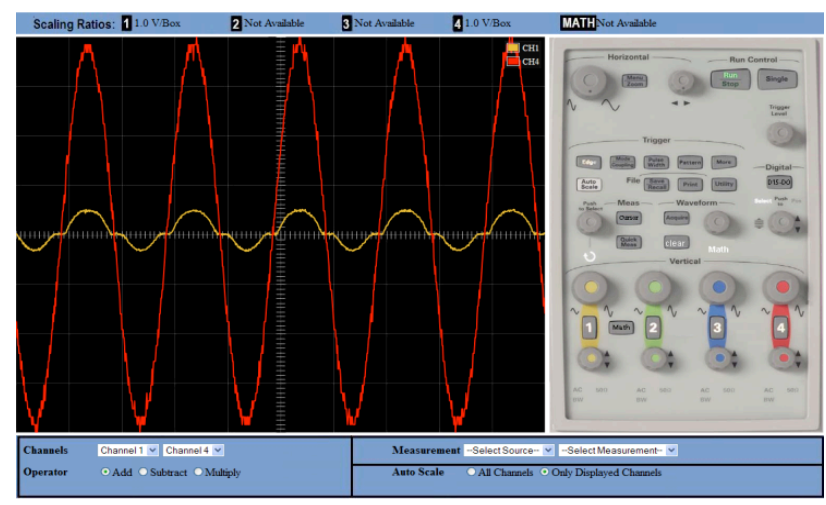

Figure 3. Design of animated oscilloscope

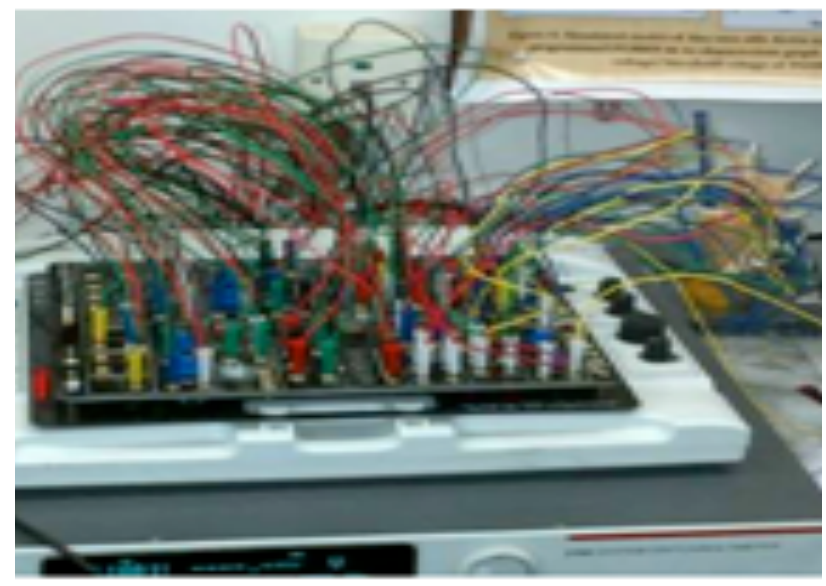

Figure 4. Hardware Setup of remote lab for analog communication electronics 


\section{B. LAN extension for Instrumentation (LXI)}

Most instrument interface standards such as GPIB, PCI, PCI-X or USB need specialized physical ports, drivers and interfacing software before any communication is possible with an instrument. In contrast, LAN based instruments are neither localized nor limited by number of ports on computer. Although Ethernet has been used in this role for quite some time but the problem was that there was no standard. The way instruments communicated over Ethernet because of interface parameters varied from one instrument to the next. It is this problem that LXI seeks to address. LXI sets standards for instruments to have an embedded Web-server, a SCPI language compiler and conformation to IVI and VISA standards. We have utilized Agilent's LXI c class based switching matrix with EMONA-DATEx to map the virtual connections on to the physical hardware.

\section{NI-ELVIS II}

National Instruments Education Laboratory Virtual Instrumentation Suite (Ni-Elvis II) consists of LabVIEW based virtual instruments and a multifunction data acquisition (DAQ) device. We have used Ni-Elvis instruments multimeter, analog two channel oscilloscope, function generator, variable power supply and digital signal analyzer. Ni-Elvis web server interface allows each instrument to be readily controlled and observed from the web on real-time basis. The data acquisition interface allows students to make real-time data acquisitions on the web. When an instrument is requested by clicking on specified button in the GUI, a window pops up showing instrument controls and the reading of the instrument as shown in Figure 5.The instrument settings can be changed by the control knobs and buttons on the client computer screen.

\section{Virtualization Software}

The virtualization software is implemented in HTML, JavaScript, Java, Visual C, LabVIEW, Shell script. Perl has been used for the CGI interface. HTML, JavaScript and the Java code resides on the main server. Elvis LabVIEW web server resides on a windows system connected to DATEx-Elvis II set up. LabVIEW web server provides NI-Elvis instrument panels (as shown in Figure5) to remote clients. Microsoft Visual Studio that provides a platform to run LabVIEW built DLL files also resides on the windows system. A Cygwin environment has been set up for interfacing the windows system with the main Linux server to acquire and send data between main server and data acquisition board (DATEx-Elvis II).

The Web-based software (written in HTML, JavaScript, LabVIEW and Java), integrates the virtual breadboard, components, scope, Emona-DATEx controllable knobs and waveform generator (customized in LabVIEW). Figure6 shows a circuit wired on the virtual Emona DATEx board just like any student will do in a traditional laboratory.

\section{E. Frontend GUI (Virtual DATEx Breadboard)}

Teleoperation of instruments placed in a physical laboratory requires a user interface that provides the user look and feel of a real instrument. It requires interactive panels and strong backend technique to be utilized to build a complete framework. These services can attract students and motivate them to use remote laboratories. With the current technologies available, it has become quite easy to remotely operate physical instruments over internet. Students are able to assemble and wire up a circuit from their computer just like they do in a traditional laboratory [9]. Providing accessibility to Emona-DATEx and NiElvis remotely with flexibility to wire up a circuit by patching up DATEx blocks has been made possible using an Agilent switching Matrix and a Web-based GUI for Emona DATEx. Figure 6 shows a typical Web-based "Emona DATEx Virtual Board".

The user can patch up any DATEx blocks over the DATEx virtual breadboard as required to accomplish the necessary wiring. When the user completes the circuit and presses the "run" button, the software at the host analyzes the circuit to extract a net-list i.e. determines which hardware pins are connected together. Figure 7 shows the connection diagram of DATEx-Elvis II set up.

The pin connections represent nodes. The circuit may be wired on any portion of the DATEx virtual breadboard. If the user mistakenly connects extra wires somewhere, the software turns on only the appropriate relays to connect the circuit wired on the DATEx virtual breadboard.
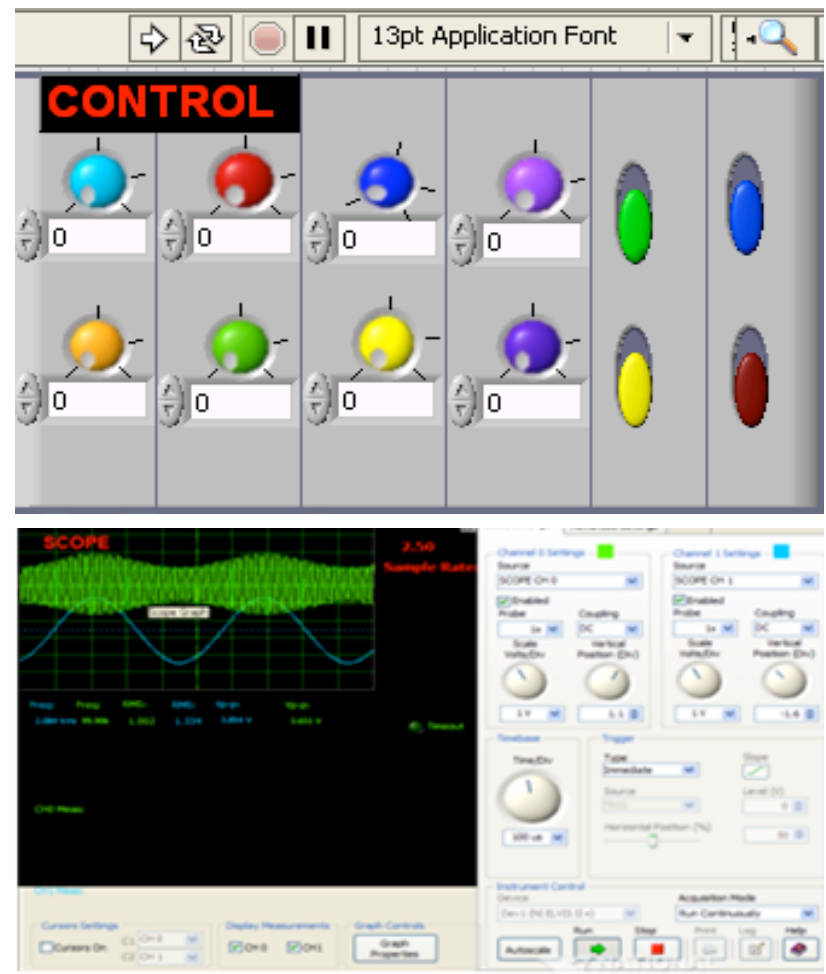

Figure 5. Upper image shows the control panel for DATEx blocks and lower image shows the Elvis oscilloscope

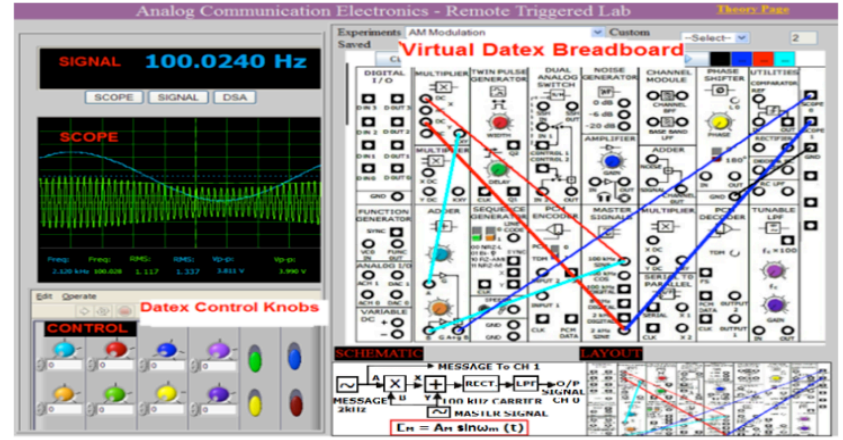

Figure 6. Webpage of remote laboratory for analog communication 
PAPER

REMOTE LABORATORIES - A ClOUd BASED MODEL FOR TELEOPERATION OF REAL LABORATORIES

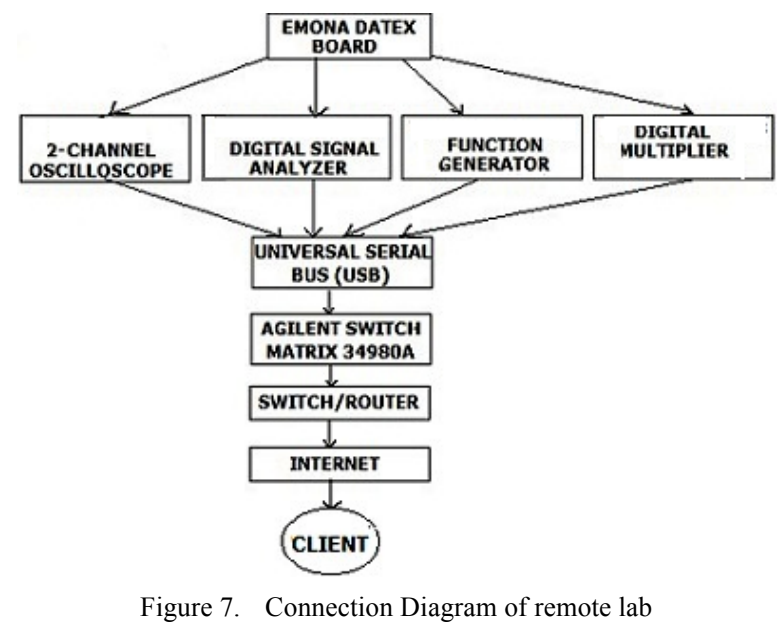

The web-page appears like a dashboard that shows a summarized view of all involved instruments and a virtual DATEx breadboard. The representative buttons provide a way to open up elaborate front panels of the measurement instruments, while the display panels of these instruments convey essential information for quick view. Background software ensures electrical rules and breadboard topology are encoded so as to extract an electrical circuit from the virtual DATEx breadboard. The user interface tries to replicate the real life experience in many ways. A student trained on these virtual panels is not likely to feel out of place in a real laboratory.

Thus, by closing specific relays, selected devices can be connected in any desired topology, thus matching the circuit to be drawn.

\section{BACKEND FLOW}

The circuit wiring tool has two primary components (i) Virtual DATEx Board and (ii) the relay matrix. The virtual DATEx board is a java program that allows the user to join the various circuit blocks which results in a netlist. The relay matrix containing fixed devices and customized relays provides realization of the circuit netlist.

This section describes the backend flow where a scheme drawn on the virtual DATEx board is converted into a list of relay IDs in order to realize the circuit.

The virtual DATEx board: user draws a scheme on the virtual DATEx board. This scheme consists of DATEx blocks and wires drawn on the board. Figure 6 shows a sample scheme on the virtual DATEx board. To infer connectivity from this scheme and create the same connectivity physically on the hardware there is a need of closing specific relays on the relay matrix.

The relay matrix: The relay matrix is a LXI switch matrix device. Typically it has 16 rows and 32 columns or a similar combination. The DATEx blocks to be used in experiments along with instruments are put on the columns. Rows and columns form a grid and each point on the grid (row, column) has a relay. The rows are not connected to the columns but any point can be shorted by closing its relay. Thus by closing specific relays, selected devices can be connected in any desired topology, thus matching the circuit to be drawn. A relay matrix containing few blocks is shown in Figure8.

At present in this lab one can wire up a circuit up to 16 nodes and 32 points. Various blocks that can be used to create complex circuits are Digital and Analog I/O, Function Generator, power supply, 3-Multiplier, Adder, Twin pulse generator, sequence generator, dual analog switch, PCM encoder and decoder, noise, amplifier, master signals, channel modules, sample and hold, phase shifter, comparator, Rectifiers and tunable LPF. The complexity of a circuit matrix increases with the number of circuit nodes. If a circuit has $\mathrm{N}$ nodes and if the user wishes to add a 2-point block, there are $\mathrm{Nx}(\mathrm{N}-1) / 2$ branches to which it can be introduced. This ensures that the numbers of devices used are limited by the size of the relay matrix.

The blocks are connected on the relay matrix once and do not change. For the relay matrix, two configuration files are hand-created as described below. These configuration files play a major role in the netlist translation.

The relay configuration file: Each relay in the relay matrix is recognized by a relay ID. The relay configuration file contains a mapping for each (row, column) to its relay ID. The relay configuration file helps the system to remain independent of the underlying relay matrix hardware. If the hardware changes or the relay mapping changes, only relay configuration needs to be updated.

The board configuration file: Information about the devices and their placements on the columns is recorded in a configuration file. The board configuration file contains information about the device, its parameters and the column numbers where the device is connected.

Conversion steps from circuit scheme to realized circuit:

1. Extraction of netlist from user's scheme, output is a text-based SPICE like netlist.

2. Mapping of netlist DATEx blocks to the corresponding block on the virtual DATEx board, output is a text-based list of (row, column).

3. Mapping of (row, column) to the relay ID list, output is a text-based list of relay IDs to close.

4. Reset relay matrix and close only those relays obtained in the step 3, results in a realized circuit on the relay matrix. This entire process is shown in the flow chart of Figure 8.

\section{SUSTAINABILITY AND SCALABILITY}

In initial stage, every technology faces difficulties and challenges and requires a great effort to replicate and replace the other. There are several factors e.g. feasibility, sustainability, affordability etc. that need to be taken into consideration at the time of implementing the technology. Once the technology has evolved and perfectly matured, the primary concern that remains is sustainability. Remote laboratories use high cost instrumentation which are more precise than physical laboratory instruments. To justify the cost of the expensive instrumentation used to build remote labs, multiuser scalability is necessary. We have introduced a queuing mechanism to process requests on first come first serve basis in quick sequential steps seen as rapid switching of the relays on the hardware. The average hardware verification time for each user is nearly 2-3 seconds. Under ordinary circumstances, it is unlikely that all users will request hardware verification simultaneously, even if such a situation arises, each user's hardware request is attended to almost immediately within seconds. 


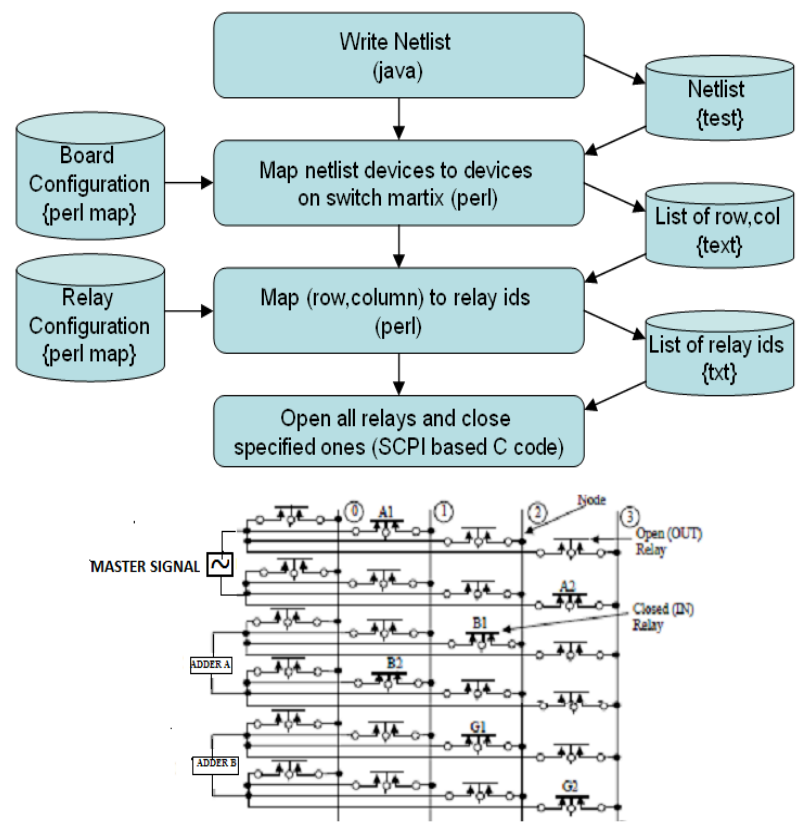

Figure 8. Flowchart showing the complete backend flow (top) shows various algorithmic steps and their interdependencies that convert a breadboard circuit into a netlist and finally into list of relays that needs to be closed to create a physical equivalent of the virtual circuit. (bottom) shows a reduced view of the switching matrix loaded with devices. The embedded web-controller provides the signals to switch the relays $\mathrm{ON}$ and $\mathrm{OFF}$.

\section{COMPARISON OF EXPERIMENTAL DATA}

In analog communication, even a small noise can affect the output of the experiment adversely. We use a switching matrix for wiring up multiple circuits and opening and closing connections in real time. So there is always a possibility for errors and interference due to medium between switching matrix and breadboard. There are several factors that can induce noise in the signal e.g. environmental disturbances, quality of wires etc. Therefore to verify that the experimental results obtained from remote lab are authentic we need to compare them with results obtained in physical lab.

To verify the experimental results and data of remote laboratory, we performed Double Sideband Suppressed Carrier (DSB-SC) Experiment (with variable frequency, variable amplitude message signal and $1 \mathrm{Vpp}, 100 \mathrm{kHz}$ carrier signal) and analyzed the experimental results obtained using remote lab. We repeated the same experiment in the physical lab (without switch matrix) and verified the results obtained in both environments. Total Harmonic Distortion (THD) was zero in both the cases but slight variation in Signal-to-Noise Distortion Ratio (SINAD) was observed as shown in Table I. Values corresponding to row (A) represents the SINAD with remote lab while row (B) represents the SINAD that corresponds to physical lab.

Figure 9 shows the 3-D comparison of SINAD in both the environments. Figure 10(a) shows the message signal and DSB-SC signal in the physical lab. Figure 10(c) shows the corresponding FFT of DSB-SC Signal having two frequency bands, SINAD value in $\mathrm{dB}$ and THD. Figure 10(b) shows the message signal and DSB-SC signal on remote lab and Figure $10(d)$ shows the corresponding FFT of DSB-SC signal having two frequency bands, SINAD value in $\mathrm{dB}$ and also THD.

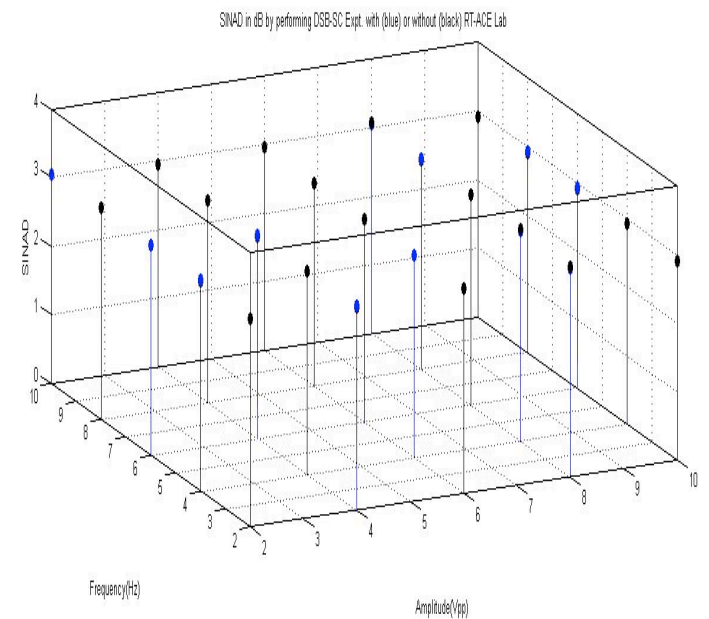

Figure 9. Comparison of SINAD in $\mathrm{dB}$ by performing DSB-SC experiment using remote lab (blue) and (black) using physical Lab

TABLE I.

SINAD IN DB BY PERFORMING DSB-SC EXPERIMENT (A) USING REMOTE LAB \& (B) USING PHYSICAL LAB

\begin{tabular}{|c|c|c|c|c|c|c|}
\hline \multicolumn{2}{|c|}{ Amplitude (Vpp) } & 2 & 4 & 6 & 8 & 10 \\
\cline { 1 - 5 } \multicolumn{2}{|c|}{ Frequency $(\mathrm{Hz})$} & & & & & \\
\hline \multirow{2}{*}{2} & A & 3.05 & 2.98 & 2.99 & 3.05 & 2.91 \\
\cline { 2 - 6 } & B & 3.05 & 2.96 & 2.99 & 3.06 & 2.91 \\
\hline \multirow{2}{*}{4} & A & 3.08 & 2.96 & 2.96 & 3.07 & 2.92 \\
\cline { 2 - 6 } & B & 3.07 & 2.96 & 2.95 & 3.08 & 2.93 \\
\hline \multirow{2}{*}{6} & A & 3.07 & 2.97 & 2.95 & 3.08 & 2.93 \\
\hline \multirow{2}{*}{8} & B & 3.06 & 2.95 & 2.96 & 3.08 & 2.91 \\
\hline \multirow{2}{*}{10} & A & 3.08 & 2.96 & 2.95 & 3.07 & 2.93 \\
\hline & B & 3.08 & 2.96 & 2.95 & 3.06 & 2.92 \\
\hline & A & 3.06 & 2.95 & 2.96 & 3.06 & 2.92 \\
\hline & B & 3.05 & 2.95 & 2.96 & 3.07 & 2.92 \\
\hline
\end{tabular}

After making comparison between the results in both the environments we found that Signal-to-Noise Distortion Ratio is almost similar in both the cases. Therefore, we can conclude that performing experiments on remote lab does not add any additional noise to the output of experiment. Hence, these results lend support of using remote labs for e-Learning environment.

\section{COMPARSION WITH OTHER REMOTE LABS}

Table II shows the comparison between our remote lab and other remote labs like NetLab and VISIR and iLabs. These labs are based on analog electronics circuits excluding iLabs that host several other remote labs. To perform this comparison, iLabs [12] based on NI-Elvis has been analyzed. Following characteristics of these labs have been analyzed.

Analog Communication: Providing accessibility to analog communication experiments.

Circuit-wiring tool: The user can wire up arbitrary circuit using given components/blocks.

Switch Matrix: The switch matrix that is controlled using software and wires up circuits on breadboard by opening and closing connections. 
Multi-User: Multi-user means whether more than one user can perform experiment at a time or not.

Instrument Control: Protocols used to communicate with instruments.

User Interface: Technologies used to develop user interface and design of instrument panels.

Supplementary material: Providing theoretical concepts of respective experiments to the students.

Block-Diagram Approach: Analog communication concepts can be well understood in block diagram approach as given in text books.

Client end Software: Software that is required on client end to control instrument panels.

\section{CONCLUSION}

As compared to traditional e-contents like virtual classrooms, e-projects etc remote laboratory conception implies additional difficulties. One major challenge is to be able to provide touch and feel of a real lab. The user must be given the ownership of the hardware where he/she can design and execute an experiment of choice that includes all the pitfalls of real lab experiment i.e. making wrong wiring, debugging a circuit and feeling of jubilation at overcoming challenges. In this work, we presented a setup that can be used to perform Analog Communication experiments remotely.

Current remote lab set up is a scalable model of $\mathrm{Ni}$ Elvis and Emona DATEx and provides a pedagogically enhanced environment for students to perform analog communication experiments using a superior block diagram approach.

\section{ACKNOWLEDGMENT}

The author C.M.Markan acknowledges Ministry of Human Resource and Development (MHRD) Government of India for the financial support under NMEICT Nationally Coordinated Project on Virtual Labs.

\section{REFERENCES}

[1] J. Biggs, Teaching for Quality Learning at University. Maidenhead, U.K.: Open Univ. Press/McGraw-Hill, 2003.

[2] S. Ursulet and D. Gillet, "Introducing flexibility in traditional engineering education by providing dedicated on-line experimentation and tutoring resources", the International Conference onEngineering Education, Manchester, UK, August 18-21, 2002

[3] N. Ertugrul, "New area in engineering experiments: An integrated and interactive/learning approach, and real-time visualizations," Int. J. Eng.Educ., vol. 14, no. 5, pp. 344-355, 1998.

[4] Auer M., Pester A., Ursutiu D., and Samoila C., "Distributed Virtual and Remote Labs in Engineering", Proc. of the ICIT'03 Intl. Conference on Industrial Technology, Maribor, Slovenia, December $10-12,2003$.

[5] Callaghan, MJ. Harkin, J., McGinnity, TM. and Maguire, LP.,"Paradigms in Remote Experimentation", iJOE(Intl.J. of Online Engg). ISSN: 1861-2121- Vol. 3, No. 4, Pg5-14, 2007

[6] Harvard V., Alamo J.D, et.al. "ilab: A scalable architecture for sharing onlineexperiments"., International Conf. on Engineering Education(ICEE2004), Gainesville, Florida, , Oct 16-21, 2004.

[7] Markan, CM., Bhatnagar, A., Gupta, A.," A Remote Triggered Electronics Laboratory using LXI methodology", Proc.of Intl. ConferenceonRemoteEngineering and Virtual Instrumentation, (REV-2010) Stockholm,June29-July 2, 2010.

[8] Akram Abu-aisheh,Farid Farahmand,Implementation of a Remote Analog and Digital Communications Laboratory for eLearning, $10^{\text {th }}$ IEEE International Conference on Advanced Learning Technologies.
TABLE II.

COMPARISON BETWEEN OUR REMOTE LAB AND OTHER REMOTE LABS

\begin{tabular}{|c|c|c|c|c|}
\hline & Our lab & iLab & $\begin{array}{c}\text { NetLab } \\
\text { (available } \\
\text { for Basic } \\
\text { Electronics) }\end{array}$ & VISIR \\
\hline $\begin{array}{c}\text { Analog Com- } \\
\text { munication }\end{array}$ & Yes & Yes & No & No \\
\hline $\begin{array}{c}\text { Circuit-wiring } \\
\text { tool }\end{array}$ & Yes & No & Yes & Yes \\
\hline Switch-Matrix & $\begin{array}{l}\text { Agilent } \\
34980 \mathrm{~A}\end{array}$ & \begin{tabular}{|c|} 
NISCZI \\
1169,100 \\
Switch [18 \\
$29], 4$ slot \\
\end{tabular} & $\begin{array}{l}\text { Agilent } \\
\text { E1465A }\end{array}$ & $\begin{array}{c}\text { Owner } \\
\text { switching } \\
\text { matrix }\end{array}$ \\
\hline Multi-User & Yes & No & Max. 3 & No \\
\hline $\begin{array}{c}\text { Instrument } \\
\text { Control }\end{array}$ & $\begin{array}{c}\text { GPIB, LXI, } \\
\text { Ni-Elvis }\end{array}$ & $\begin{array}{c}\text { GPIB, Ni- } \\
\text { Elvis }\end{array}$ & GPIB-VXI & PXI \\
\hline User Interface & $\begin{array}{c}\text { Virtual } \\
\text { Breadboard } \\
\text { in java and } \\
\text { interactive } \\
\text { instrument } \\
\text { panels } \\
\end{array}$ & $\begin{array}{c}\text { Java } \\
\text { applet }\end{array}$ & $\begin{array}{l}\text { Virtual } \\
\text { Breadboard } \\
\text { in java }\end{array}$ & $\begin{array}{l}\text { Virtual } \\
\text { Breadboard } \\
\text { in flash }\end{array}$ \\
\hline $\begin{array}{c}\text { Supplementary } \\
\text { material }\end{array}$ & Yes & No & No & No \\
\hline $\begin{array}{c}\text { Block-Diagram } \\
\text { Approach }\end{array}$ & Yes & Yes & No & No \\
\hline $\begin{array}{l}\text { Client end } \\
\text { Software }\end{array}$ & $\begin{array}{l}\text { Java and } \\
\text { Labview*** }\end{array}$ & LabVIEW & Java & LabVIEW \\
\hline
\end{tabular}

***In interactive mode Labview is required, while in batch mode LabVIEW is not necessary.
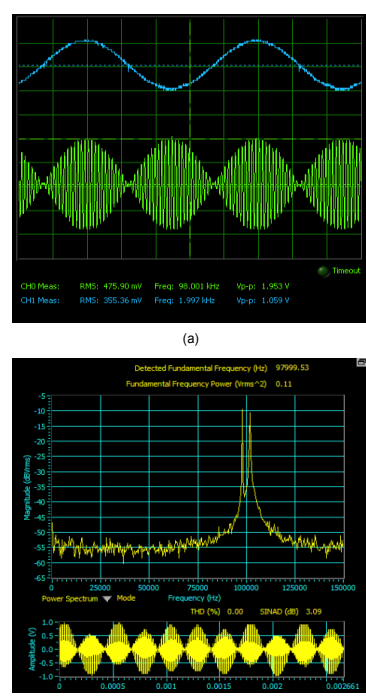

(b)
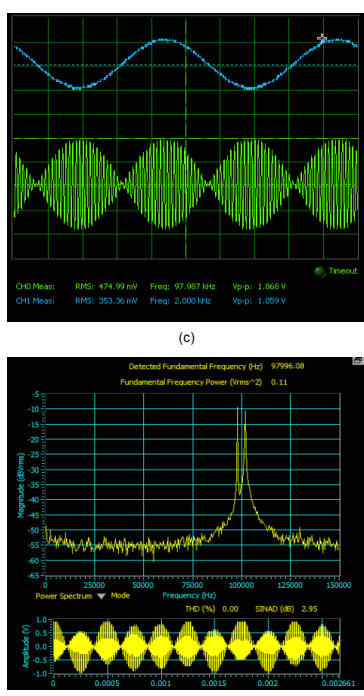

Figure 10. (a) Shows the Message and DSB-SC signal without using remote lab, (b) Shows the Message and DSB-SC signal by using remote lab, (c) FFT of DSB-SC Signal obtained using physical lab, (d) FFT of DSB-SC Signal obtained by using remote lab

[9] C.M.Markan, Priti Gupta, Manas, Goutam Kumar, Satendra Gupta, Scalable Multiuser Remote Laboratories provide ondemand hands-on laboratory experience,Remote Labs session, IEEE 2012 Conferenceon Technology and Society in Asia, Singapore Oct27-29, 2012

[10] Fujii N and Koike N, "A Dual Mode Remote Laboratory SystemSupporting Both Real-Time and Batch Controls by MakingUse of Virtual Machines", 38th ASEE/IEEE Frontiers in Education Conference, Saratoga Springs, NY, October 22 - 25, 2008 ,

[11] Akram Abu-aisheh, Tom Eppes, Abdullah Y Alzoubi,'Implementation of a Remote Analog and Digital Commu- 
nicationsLaboratory for e-Learning",Internation Journal of OnlineEngineering (IJOE), vol.6,no.2,2010

[12] Helen Kyomugisha, Tom Kigezi, Cosmas Mwikirize, Roseline Akol, Doreen Orishaba, Michael Kyesswa, "A Remote Direct Sequence Spread SpectrumCommuniocations Lab Utilising the Emona DATEx “ Makerere University, Kampala- Uganda, Internationa Journal of Online Engineering (IJOE), vol.8,2012

[13] I Gustavsson, T Olsson, H Akesson, J Zakrisson, L Hakansson “ A Remote Electronics Laboratory for Physical Experiments Using Virtual Breadboards",proceeding of the 2005 ASEE Annual Conference, Portland, USA, june 12- 15, 2005

[14] Hamadou Saliah-Hassane,Maarouf Saad, Willie K.Ofosu, Karimou djibo, Hassane Alzouma Mayaki, Mamane Moustapha Dodo Amadou, "Lab@Home :Remote Laboratory Evolution in cloud computing era " AC 2011:2530

[15] Z. Nedic and J. Machotka,"Remote Laboratory NetLab for Effective Teaching of 1 Year Engineering Students*" Internation Journal of Online Engineering (IJOE), vol.3,no.3,2007

[16] J. García-Zubia, U. Hernández, I. Angulo, P. Orduña, J. IrurzunUniversity of Deusto, Bilbao, Spain,"Remote Laboratories Based on LXI" Internation Journal of Online Engineering (IJOE), vol.4,no.3,2008

[17] L. Claesson and L. HåkanssonBlekinge Institute ofTechnology,Karlskrona, Sweden, "Using an Online RemoteLaboratory for Electrical Experiments in Upper SecondaryEducation" International Journal of Online Engineering(IJOE)-vol 8,special issue:2 “exp .at'11",2012

[18] Kigezi, T. ; Mwikirize, C. ; Akol, R. ; Orishaba, D. ; Kyesswa, M. "A remote direct sequence spread spectrum communications lab utilising the Emona DATEx "Remote Engineering and Virtual Instrumentation(REV), 2012

[19] Zutin, Danilo Garbi Auer,Michael E. Gustavsson,Ingvar,"A Visir Labserver for Ilab Shared Architecture", IEEE EDUCON,2011

[20] del Alamo, J.A. Lerman, S.R. Bailey, P.H. Carpenter, J. DeLong, K. Felknor, C. Hardison, J. Harrison, B. Jabbour, I. Long, P.D. Tingting Mao Naamani, L. Northridge, J. Schulz, M. Talavera, D. Varadharajan, C. Shaomin Wang Yehia, K. Zbib, R. Zych, D. ," iLab Shared Architecture: A Web Services Infrastructure to Build
Communities of Internet Accessible Laboratories" Proceedings of IEEE, vol-96, issue-6,2008

[21] Maria T. Restivo, Member, IEEE, Joaquim Mendes, António M. Lopes, Carlos M. Silva, and Fátima Chouzal, "A Remote Laboratory in Engineering Measurement" IEEE Transactions on Industrial Electronics Vol. 56 No. 12 December 2009

[22] Petros J. Axaopoulos, Konstantinos N. Moutsopoulosa \& Michael P. Theodoridis," Engineering Education Using a Remote Laboratory through the Internet", European Journal of Engineering Education, v37 n1 p39-48 2012

\section{AUTHORS}

Dr. C. M. Markan is an Associate Professor in Department of Physics and Computer Science, Dayalbagh Educational Institute, Dayalbagh, Agra, India. His areas of interests are in Neuromorphic and Adaptable Analog VLSI, Virtual and Remote Laboratories. He is a member of IEEE, Systems Society of India, and International Association of Online Engineering (cm.markan@gmail.com)

Satendra Gupta is with the Department of Physics \& Computer Science, Dayalbagh Educational Institute, Agra, India (sunny.shal.gupta@gmail.com)

Sajal Mittal is a Graduate Student in Faculty of Electrical Engineering, Indian Institute of Technology, Kanpur. He did his under graduation from Electrical Department, Dayalbagh Educational Institute. $\mathrm{He}$ is student member of IEEE Society and actively participating in its activities (sajalmitta182@yahoo.co.in)

Goutam Kumar is a software developer in MHRD project in Dayalbagh Educational Institute, Dayalbagh, Agra, India. His interests are programming in java and web application development. (goutamns@gmail.com)

Received 26 January 2013. Published as resubmitted by the authors 25 April 2013. 